\title{
Sequenztherapie beim fortgeschrittenen Nierenzellkarzinom
}

\author{
Interview mit PD Dr. Dirk Arnold, Universitätsklinikum Halle/Saale
}

Everolimus (Afinitor ${ }^{\oplus}$ ) wurde 2009 zugelassen zur Behandlung von Patienten mit fortgeschrittenem Nierenzellkarzinom, bei denen es während oder nach der Therapie mit einem TyrosinkinaseInhibitor zu einer Progression kommt. Welche Bedeutung hat die Zulassung von Everolimus für die Therapie des fortgeschrittenen Nierenzellkarzinoms?

Mit Everolimus steht nun die fünfte zielgerichtete Substanz für die Therapie des fortgeschrittenen Nierenzellkarzinoms zur Verfügung. Der oral verfügbare mTOR (mammalian Target of Rapamycin)-Inhibitor spielt eine wichtige Rolle in der sequenziellen Therapieabfolge, die heute Standard ist. First-line werden meist gegen VEGF (Vascular Endothelial Growth Factor) gerichtete Therapien wie der Tyrosinkinase-Inhibitor Sunitinib oder der VEGF-Antikörper Bevacizumab - dieser in Kombination mit Interferon alfa - eingesetzt. Zur Therapie vorbehandelter, d.h. meist refraktärer Patienten oder bei Patienten mit Unverträglichkeiten, kann mit diesem mTORInhibitor nun eine Substanz mit anderem Wirkungsmechanismus genutzt werden. Bis zu seiner Zulassung konnten Patienten mit einer Krankheitsprogression unter der ersten gegen VEGF-gerichteten Therapie nur mit einem (anderen) Tyrosinkinase-Inhibitor behandelt werden. Wir haben jetzt mit Everolimus eine Alternative für die Folgetherapie.

\section{Wann setzen Sie Everolimus ein?}

Die individuelle Planung des Therapiemanagements ist wichtig. Denn jeder Patient hat einen anderen Krankheitsverlauf, und auch die Nebenwirkungen können stark differieren. Bei fast allen unserer Patienten wurde first-line eine gegen VEGF-gerichtete Therapie eingesetzt. Daher erfolgt der Einsatz von Everolimus bei uns häufig direkt nach Progression unter der ersten Tyrosinkinase-Inhibitor-Therapie. Aus Mangel an Alternativen hat sich vor der EverolimusZulassung im Klinikalltag die Sequenz der beiden Tyrosinkinase-Inhibitoren, Sunitinib gefolgt von Sorafenib, durchgesetzt. Auch bei den so vorbehandelten Patienten wenden wir Everolimus an, dann gewissermaßen als Third-line-Therapie, oder fourthline, wenn mit Interferon alfa und Bevacizumab begonnen worden war.

Die bessere Evidenz besteht aber für den Einsatz des mTOR-Inhibitors nach Krankheitsprogression unter der ersten Tyrosinkinase-Inhibitor-Therapie: In der RECORD-1-Studie wurde bei Behandlung mit Everolimus nach Krankheitsprogression unter Tyrosinkinase-Inhibitor-Therapie ein signifikant längeres progressionsfreies Überleben im Vergleich zu Placebo gezeigt (median 1,9 Monate vs. 4,9 Monate; p<0,001; Motzer RJ et al., 2008, Lancet 372: 449-456 [Update ASCO-GU 2009]). Die bislang vorliegenden Praxiserfahrungen bestätigen die Ergebnisse dieser Studie. In internationalen Therapieleitlinien wird der Einsatz dieses mTOR-Inhibitors nach der Therapie mit dem ersten Tyrosinkinase-Inhibitor empfohlen (NCCN Clinical Practice Guidelines in Oncology ${ }^{\mathrm{TM}}$, Kidney Cancer V.2.2010).

\section{Was spricht für die Behandlung mit} Everolimus nach Progression unter dem ersten Tyrosinkinase-Inhibitor?

Nach Progression unter Tyrosinkinase-Inhibitoren spricht in erster Linie der andere Wirkungsmechanismus für den Einsatz von Everolimus. Tyrosinkinase-Inhibitoren hemmen gezielt die intrazellulären Tyrosinkinase-Domänen von WachstumsfaktorRezeptoren, wie z.B. dem VEGF-Receptor oder dem Platelet Derived Growth Factor Receptor. Sie blockieren, trotz einiger Unterschiede im Affinitätsprofil, vergleichbare "Targets" - und letztendlich sehr ähnliche Signalwege.

Der mTOR-Inhibitor Everolimus interagiert mit anderen Signalwegen: Er hemmt gezielt die Serin/Threonin-Kinase mTOR. Diese Kinase wirkt als zentraler Regulator von Tumorzellwachstum, -stoffwechsel sowie der Angiogenese und damit sowohl an der Tumorals auch an der Endothelzelle. Die

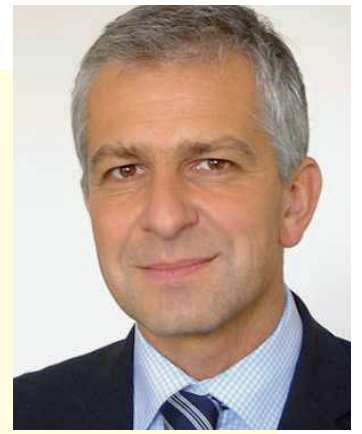

PD Dr. Dirk Arnold, Halle durch Everolimus bedingte Hemmung der mTOR-Signalkaskade, die sich „downstream" der Targets von Tyrosinkinase-Inhibitoren befindet, führt zu reduzierter Produktion von Wachstumsfaktoren, verringertem Zellwachstum und gehemmter Proliferation - bei Tumor- und Endothelzellen, Perizyten und Fibroblasten und Bone Marrow Derived Cells.

Möglicherweise können durch die mTOR-Inhibition Resistenzmechanismen überwunden werden, die sich unter der ersten antiangiogenen Therapie entwickelt haben. Allerdings wissen wir über die zellulär ablaufenden Vorgänge sehr wenig.

Welche Erfahrungen haben Sie mit Everolimus im Klinikalltag gesammelt? Wie bei jeder Therapie treten auch unter Everolimus Nebenwirkungen auf. Das $\mathrm{Ne}-$ benwirkungsprofil dieses mTOR-Inhibitors ist günstig - auch verglichen mit Nebenwirkungen anderer zielgerichteter Therapien, wie vor allem den symptomatischen Nebenwirkungen der Tyrosinkinase-Inhibitoren. Eine Nebenwirkung, die bei Anwendung von mTOR-Inhibitoren auftreten kann, ist die nicht-infektiöse Pneumonitis. Diese wird als Klasseneffekt der mTOR-Inhibitoren beschrieben. Bei den bislang bei uns etwa 20 mit Everolimus therapierten Patienten haben wir diese Nebenwirkung nicht beobachtet.

Generell führt ein kurzzeitiger Abbruch der Everolimus-Therapie bei den meisten Patienten zu einer vollständigen Heilung der nicht-infektiösen Pneumonitis. Je nach Schweregrad kann auch über einen kurzen Zeitraum die Behandlung mit Glucocorticoiden sinnvoll sein. Nach Rückgang der Pneumonitis sollte die Therapie mit einer reduzierten Everolimus-Dosierung fortgesetzt werden. 